\title{
Combining the Global and Partial Information for Distance-Based Time Series Classification and Clustering
}

\author{
Hui Zhang*, Tu Bao Ho*, Mao-Song Lin**, and Wei Huang* \\ * School of Knowledge Science, Japan Advanced Institute of Science and Technology \\ Nomi, Ishikawa 923-1292, Japan \\ E-mail: \{zhang-h, bao,w-huang\}@ jaist.ac.jp \\ ** School of Computer Science, Southwest University of Science and Technology \\ Mianyang, Sichuan 621002, China \\ E-mail: 1ms@swust.edu.cn \\ [Received March 23, 2005; accepted August 11, 2005]
}

\begin{abstract}
Many time series representation schemes for classification and clustering have been proposed. Most of the proposed representation focuses on the prominent series by considering the global information of the time series. The partial information of time series that indicates the local change of time series is often ignored. Recently, researches shown that the partial information is also important for time series mining. However, the combination of these two types of information has not been well studied in the literature. Moreover, most of the proposed time series representation requires predefined parameters. The classification and clustering results are considerably influenced by the parameter settings, and, users often have difficulty in determining the parameters.

We attack above two problems by exploiting the multiscale property of wavelet decomposition. The main contributions of this work are: (1) extracting features combining the global information and partial information of time series (2) automatically choosing appropriate features, namely, features in an appropriate wavelet decomposition scale according to the concentration of wavelet coefficients within this scale. Experiments performed on several benchmark time series datasets justify the usefulness of the proposed approach.
\end{abstract}

Keywords: time series, Haar wavelets, classification, clustering, feature extraction

\section{Introduction}

Time series data are popularly used in various domains like finance, bioinformatics, science and medical diagnosis. Many algorithms have been proposed for mining time series data $[17,28]$. Time series classification and time series clustering are two important aspects of time series mining. Time series classification has been successfully used in various applications such as medical data analysis [10,33], sign language recognition [13], speech recognition [21], etc. Time series clustering is a popularly used preprocessing technique in stock market analysis [8], gene expression data analysis [11], and so on. Most time series contain long sequences with high dimensionality, the neighbouring points within a time series have strong relationship, and normally time series are stained by noise [17]. These properties make time series classification and clustering challenging tasks. For efficiency, most of the proposed methods classify or cluster time series on the high level representation of time series that takes the global information of time series rather than classifying or clustering them directly. The representation includes Fourier Transforms [1,26], Piecewise Linear Representation (PLR) [19], Piecewise Aggregate Approximation [15, 34], Regression Tree Representation [9], Wavelets [3, 25], Singular Value Decomposition [20], and Symbolic Representation [22], etc. Recently, Jin et al. proposed a time series representation scheme based on the partial information of the time series and showed that the partial information is also important for time series mining [12]. However, to our knowledge, no work has been done to combine these two types of information in time series mining literature.

Most of the proposed representation schemes in the literature require predefined parameters. The data mining algorithms are considerably influenced by the predefined parameters [18]. This problem also holds true for time series representation, for example, when setting the number of straight lines as the input parameter for the PLR algorithm, the range of selection is limited from one to the length of raw time series data. If the input parameter is one, the representation is just a linear regression of the whole data set, and the classification accuracy in this case will be lower than using raw data for most data and algorithms. If we choose the length of raw time series data as the input parameter, the represented data is actually the raw data. The selection is not trivial or easy for the users, and as a consequence, they usually have difficulty in determining the parameters. Note that a domain-transform technique such as Fourier transform doesn't need input parameters itself, but the later feature extraction process needs input parameters in most cases. 
In this paper we introduce a time series representation combining both the global information and the partial information of the time series data by Haar wavelet decomposition. Time series classification and clustering algorithms are performed with the selected features of the representation. We propose a novel non-parametric feature extraction algorithm that extracts the approximation (global information) and change amplitudes (partial information) of time series. The Euclidean distances between the features of shifted time series and original time series are smaller than those between the raw data. Hence, distance-based classification and clustering algorithms are suitable for the features. The appropriate features, i.e., features within the appropriate wavelet decomposition scale, are chosen with respect to the concentration of features. The appropriate features are robust against noise embedded in the time series. Experiments performed on several benchmark datasets demonstrate the effectiveness of the proposed approach.

The rest of this paper is organized as follows. Section 2 briefly discusses the related work. Section 3 introduces our feature extraction algorithm and the corresponding distance-based classification and clustering algorithms. Section 4 contains an comparative experimental evaluation with the proposed approach. Finally, Section 5 concludes the paper with summarizing the main contributions of the work.

\section{Related Work}

A large number of wavelet-based feature extraction techniques have been proposed. Chan and $\mathrm{Fu}$ introduced an algorithm for nearest neighbor querying with Haar wavelet coefficients, and only first few wavelet coefficients were preserved for dimensionality reduction [3]. Popivanov and Miller used first few Daubechies wavelet coefficients instead of Haar wavelet coefficients for time series querying [25]. Shahabi et al. proposed an algorithm called TSA-tree which queries either the approximation part or detail part of Haar wavelet coefficients in a specific scale given by the user [29]. Struzik and Siebes defined a new time series similarity measurement on the correlation of Haar wavelet coefficients [31]. In the signal processing community, Piter and Kamarthi chose clustered wavelet coefficients as features [24]. Tancel et al. only used approximation wavelet coefficients as input to an ART-2 type neural network [32]. Kalayci and Ozdamar suggested a method using eight central detail coefficients of scale $m$ ranging from 1 to 5 as the features [14].

To date, all the proposed methods use global information or partial information, but no proposed algorithm combines these two types of information. Furthermore, no proposed work gives solution for automatically choosing appropriate features for a given dataset. We propose a method of using entropy to choose appropriate scale which is similar in spirit to the wavelet packet algorithm introduced by Coifman et al. [4], in which the entropy is used to select the best basis for a wavelet packet.

\section{Time Series Representation and Feature Ex- traction}

Our basic idea is to extract features from time series and perform classification and clustering algorithms with the extracted features. The multi-scale property of wavelets allows us to extract features with the global information and partial information simultaneously. After obtaining the features, distance-based classification and clustering algorithms can be applied in terms of the similarity between the extracted features. Therefore, we decompose our task into three sub-procedures: (1) representing the time series via wavelet coefficients within various scales which contain both the global and partial information of the time series data; (2) retrieving the features by selecting the appropriate scale of the representation; and (3) designing a similarity measuring strategy, in which most proposed similarity models could be applied. The basic idea of Haar wavelet decomposition is introduced in Section 3.1. We give the time series representation and corresponding feature extraction algorithms in Sections 3.2 and 3.3. Section 3.4 presents the method of noise reduction with the features. We suggest the similarity measure strategy and its corresponding classification and clustering algorithms in sections 3.5.

\subsection{Haar Wavelet Decomposition}

Wavelet transform is a domain transform technique for hierarchically decomposing a sequence from time domain to a time-frequency domain [2]. It describes a time series in terms of an approximation of the original sequence, plus a set of details that range from coarse to fine with various scales. The multi-scale property of wavelet transform allows us to observe a time series with different aspects, from very detail to very board. One property of wavelets is that the broad trend of the input sequence is preserved in the approximation part, whereas localized changes are kept in the detail parts. No information is gained or lost during the decomposition process. The original signal can be fully reconstructed from the approximation part and the detail parts.

The Haar wavelet is the simplest and most popular wavelet proposed by Haar. The benefit of the Haar wavelet is that its decomposition process has low computational complexity. Given a time series with length $n$, where $n$ is an integral power of 2, the complexity of Haar decomposition is $O(n)$ [3]. The Haar wavelet decomposition process needs a pair of sequences associated with it. The sequences are called wavelet analysis filters, denoted as $\left\{h_{k}, g_{k}\right\}$. The Haar wavelet analysis filters $h_{k}$ and $g_{k}$ are given by

$$
h_{k}=[1 / \sqrt{2}, 1 / \sqrt{2}] \text { and } g_{k}=[1 / \sqrt{2},-1 / \sqrt{2}] .
$$

Haar wavelet decomposition can be implemented by a two-step process: down-sampling and a convolution with the down-sampled series and wavelet analysis filters. The length of the input time series is restricted to an integer power of 2 in the process of wavelet decomposition. 
The series will be extended to an integer power of 2 by padding zeros to the end of time series if the length of the input time series doesn't satisfy this requirement.

A time series $\vec{X}=\left\{x_{1}, x_{2}, \ldots, x_{n}\right\}$ can be decomposed into an approximation part $\overrightarrow{A_{1}}=\left\{\left(x_{1}+x_{2}\right) / \sqrt{2},\left(x_{3}+\right.\right.$ $\left.\left.x_{4}\right) / \sqrt{2}, \ldots,\left(x_{n-1}+x_{n}\right) / \sqrt{2}\right\}$ and a detail part $\overrightarrow{D_{1}}=$ $\left\{\left(x_{1}-x_{2}\right) / \sqrt{2},\left(x_{3}-x_{4}\right) / \sqrt{2}, \ldots,\left(x_{n-1}-x_{n}\right) / \sqrt{2}\right\}$. The $\overrightarrow{A_{1}}$ are approximation coefficients within scale 1 and $\overrightarrow{D_{1}}$ are detail coefficients within scale 1 . The approximation coefficients and detail coefficients within a particular scale $j, \overrightarrow{A_{j}}$ and $\overrightarrow{D_{j}}$, both having length $n / 2^{j}$, can be decomposed from $\overrightarrow{A_{j-1}}$, the approximation coefficients within scale $j-1$ recursively. The $i$ th element of $\overrightarrow{A_{j}}$ is calculated as

$$
a_{j}^{i}=\frac{1}{\sqrt{2}}\left(a_{j-1}^{2 i-1}+a_{j-1}^{2 i}\right), \quad i \in\left[1,2, \ldots, n / 2^{j}\right] .
$$

The $i$ th element of $\overrightarrow{D_{j}}$ is calculated as:

$$
d_{j}^{i}=\frac{1}{\sqrt{2}}\left(a_{j-1}^{2 i-1}-a_{j-1}^{2 i}\right), \quad i \in\left[1,2, \ldots, n / 2^{j}\right] .
$$

The number of decomposing scales for $\vec{X}$ is $J=$ $\log _{2}(n) . \overrightarrow{A_{J}}$ only has one element denoting the global average of $\vec{X}$.

The reconstruction algorithm just is the reverse process of decomposition. The $\overrightarrow{A_{j-1}}$ can be reconstructed by formulas (3) and (4).

$$
\begin{aligned}
& a_{j-1}^{2 i-1}=\frac{1}{\sqrt{2}}\left(a_{j}^{i}+d_{j}^{i}\right), \quad i \in\left[1,2, \ldots, n / 2^{j}\right] . . \\
& a_{j-1}^{2 i}=\frac{1}{\sqrt{2}}\left(a_{j}^{i}-d_{j}^{i}\right), \quad i \in\left[1,2, \ldots, n / 2^{j}\right] . .
\end{aligned}
$$

\subsection{Time Series Representation and Feature Ex- traction}

The concatenation of decomposed wavelet coefficients of a time series $\vec{X}=\left\{x_{1}, x_{2}, \ldots, x_{n}\right\}$ to a particular scale $k \in[1,2, \ldots, J]$ shown in Eq.(5) is a representation of $\vec{X}$. An example of decomposing a time series to scale 2 is illustrated in Fig.1. The $\vec{X}$ can be fully reconstructed from $\overrightarrow{W_{k}(X)}$ without losing any information.

$$
\overrightarrow{W_{k}(X)}=\left[\overrightarrow{A_{k}(X)}, \overrightarrow{D_{k}(X)}, \ldots, \overrightarrow{D_{2}(X)}, \overrightarrow{D_{1}(X)}\right]
$$

Chan et al. proved that the Euclidean distance is preserved through a Haar wavelet transform [3]. Assume we have two time series $\vec{X}=\left\{x_{1}, x_{2}, \ldots, x_{n}\right\}$ and $\vec{Y}=$ $\left\{y_{1}, y_{2}, \ldots, y_{n}\right\}$, the Euclidean distance between $\vec{X}$ and $\vec{Y}$ is

$$
\operatorname{Disc}(\vec{X}, \vec{Y})=\sqrt{\sum_{i=1}^{n}\left(x_{i}-y_{i}\right)^{2}} \ldots . .
$$

The Euclidean distance between $\overrightarrow{W_{k}(X)}$ and $\overrightarrow{W_{k}(Y)}$, $\operatorname{Disc}\left(\overrightarrow{W_{k}(X)}, \overrightarrow{W_{k}(Y)}\right)$ is equal to $\operatorname{Disc}(\vec{X}, \vec{Y})$. In this case, if we just use $\vec{W}_{k}$ as the features for a distance based classification or clustering algorithm, the result should be the

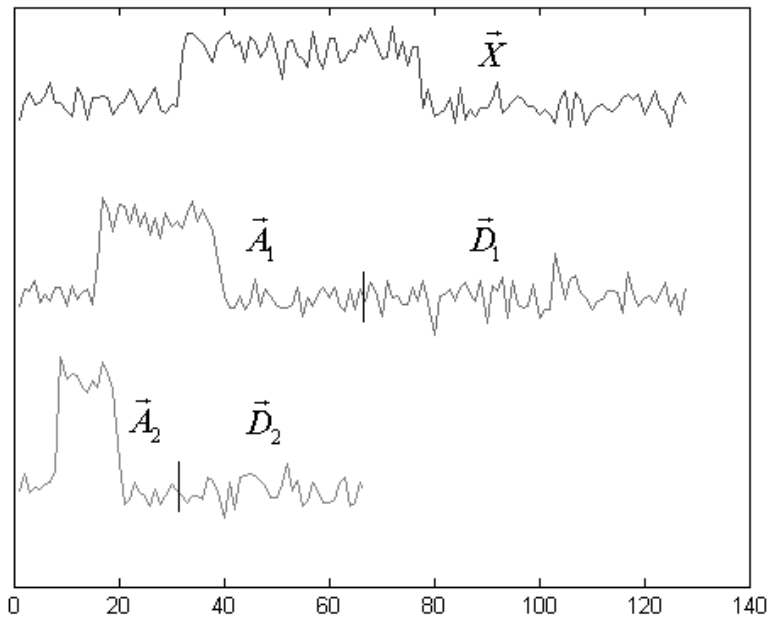

Fig. 1. An example of a time series and its wavelet coefficients to scale 2 .

same with that gotten from the original time series.

The $i$ th element of $\overrightarrow{A_{j}}$ corresponds to the segment in the series $\vec{X}$ starting from position $(i-1) * 2^{j}+1$ to position $i * 2^{j}$. The $a_{j}^{i}$ is proportional to the average of this segment and thus can be viewed as the approximation of the segment. Thus the approximation coefficients within different scales provide an understanding of the major trends in the data at a particular level of granularity. From Eq.(2), we know that the detail coefficients $\overrightarrow{D_{j}}=\left\{d_{j}^{1}, d_{j}^{2}, \ldots, d_{j}^{n / 2^{j}}\right\}$ contain local changes of time series. Thus the absolute values of the detail coefficients described in Eq.(7) denote the amplitude of local changes.

$$
\left|\overrightarrow{D_{j}}\right|=\left\{\left|d_{j}^{1}\right|,\left|d_{j}^{2}\right|, \ldots,\left|d_{j}^{n / 2^{j}}\right|\right\} \ldots . . . .
$$

We define the concatenation of decomposed wavelet approximation coefficients $\overrightarrow{A_{k}(X)}$ and the absolute values of decomposed wavelet detail coefficients $\left|\overrightarrow{D_{j}(X)}\right|, j=$ $1,2, \ldots, k$ to a particular scale $k(k \in[1,2, \ldots, J])$ of a time series $\vec{X}$ as features.

$$
\overrightarrow{F_{k}(X)}=\left[\overrightarrow{A_{k}(X)},\left|\overrightarrow{D_{k}(X)}\right|, \ldots,\left|\overrightarrow{D_{2}(X)}\right|,\left|\overrightarrow{D_{1}(X)}\right|\right] .
$$

This definition helps to overcome the well-known problem posed by the fact that wavelet coefficients are sensitive to shifts of series. The Euclidean distance $\operatorname{Disc}\left(\overrightarrow{F_{k}(X)}, \overrightarrow{F_{k}(Y)}\right)$ between the features of two time series $\vec{X}$ and $\vec{Y}$ is

$$
\sqrt{\sum_{i}\left(a_{k}^{i}(\vec{X})-a_{k}^{i}(\vec{Y})\right)^{2}+\sum_{j=1}^{k} \sum_{i}\left(\left|d_{j}^{i}(\vec{X})\right|-\left|d_{j}^{i}(\vec{Y})\right|\right)^{2}} .
$$

Because $|x|-|y| \leq x-y$, we obtain $\operatorname{Disc}\left(\overrightarrow{F_{k}(X)}, \overrightarrow{F_{k}(Y)}\right) \leq \operatorname{Disc}\left(\overrightarrow{W_{k}(X)}, \quad \overrightarrow{W_{k}(Y)}\right), \quad$ and $\operatorname{Disc}\left(\overrightarrow{W_{k}(X)}, \bar{W}_{k}(Y)\right)=\operatorname{Disc}(\vec{X}, \vec{Y})$. If $\vec{X}$ and $\vec{Y}$ denote the original time series and shifted time series respectively, this inequation is still tenable. 


\subsection{Appropriate Scale Selection}

Normally, for a time series $\vec{X}$, the first few coefficients of $\overrightarrow{W_{J}(X)}$ are taken as features after decomposing $\vec{X}$ to the scale $J[3,25]$. From Eq.(3) and Eq.(4), it's easy to observe that the first few wavelet coefficients are correspond to the approximation wavelet coefficients in lower scales including the global information of $X$. However, setting the parameter $k$ is not easy for the users and the later classification or clustering process are affected by poorly chosen parameter settings. A non-parametric feature extraction algorithm without any input parameter is more convenient to the users. For our definition of features, the nonparametric feature extraction algorithm needs to find out which features associated with a specific scale are better than others for classification and clustering automatically.

If the energy of wavelet coefficients within a scale is concentrated in a few coefficients then just those important coefficients can represent the whole, with low error. This scale may give valuable information for classification and clustering. We need a function to describe the energy concentration of the wavelet coefficients. The function should be large when coefficients are largely the same value, and small when all but a few coefficients are negligible. Shannon entropy [30], which is a measure of impurity within a set of instances satisfies our requirement, is defined as

$$
H=-\sum_{i} p_{i} \log _{2} p_{i} \ldots \ldots \ldots
$$

The appropriate decomposing scale is defined as the scale with the lowest entropy. The appropriate features of a time series are defined as the wavelet coefficients within an appropriate decomposing scale.

$$
\text { Appropriate scale }=\underset{k}{\operatorname{argmin}}\left(-\sum_{i} p_{k}^{i} \log _{2} p_{k}^{i}\right) .
$$

here $p_{k}^{i}=\left|F_{k}^{i}(X)\right| / \sum_{i=1}^{n}\left|F_{k}^{i}(X)\right|$ is the proportion between the absolute value of a coefficient in a feature and the sum of the absolute values of a whole feature. $p_{k}^{i}$ is proportional to the energy ratio of each coefficient to coefficients within a feature. $p_{k}^{i}$ has properties $\sum_{i} p_{k}^{i}=1$ and $p_{k}^{i} \geq 0$.

\subsection{Noise Reduction on the Appropriate Features}

The idea of wavelet noise reduction is based on the assumption that the amplitude of the spectra of the signal is as different as possible from that of noise [5,6]. If a signal has its energy concentrated in a small number of wavelet coefficients, these coefficients will be relatively large compared to the noise, which has its energy spread over a large number of coefficients. This allows thresholding of the amplitude of the coefficients to separate signals or remove noise. The thresholding is based on a value $\tau$ that is used to compare all the detail coefficients. Appropriate scale, as defined in section 3.3, should be robust to the noise because the energy of true signal gets concentrated in a few coefficients and the noise remains spread out in that scale. Hence the energy of the few coefficients is much larger than that of noises and these large coefficients can dominate the classification or clustering process. To verify the claim, we will compare the classification and clustering results with and without noisereduction in the experiments.

Donoho and Johnstone [6] gave the threshold as $\tau=$ $\sigma_{n} \sqrt{2 \log (N)}$; here $\sigma_{n}$ is the standard variation of noise, and $N$ is the length of the time series. Because we don't know the $\sigma_{n}$ of the time series in advance, we estimate it by the robust median estimation of noise method described in [6]. The robust median estimation is the median absolute deviation of the detail wavelet coefficients at scale one, divided by 0.6745 .

The widely used hard thresholding algorithm is a process of setting the value of detail coefficients whose absolute values are lower than the threshold to zero [5]. The hard thresholding algorithm for features defined in Eq.(11) is described in Eq.(12).

$$
\operatorname{Thre}\left(\left|d_{j}^{i}\right|\right)= \begin{cases}\left|d_{j}^{i}\right|, & \left|d_{j}^{i}\right|>\tau \\ 0, & \left|d_{j}^{i}\right| \leq \tau\end{cases}
$$

\subsection{The Similarity Strategy and its Corresponding Classification and Clustering Algorithms}

For two series with the same length, their corresponding appropriate scales may not be equal. We can't compare the similarity of two sets of appropriate features directly because the meaning of each data entry is different. For example, consider a time series $\vec{X}=\left\{x_{1}, x_{2}, x_{3}, x_{4}\right\}$ with appropriate scale 1 and a time series $\vec{Y}=\left\{y_{1}, y_{2}, y_{3}, y_{4}\right\}$ with appropriate scale 2. The features of series $\vec{X}$ are $\overrightarrow{F_{1}(X)}=$ $\left\{a_{1}^{0}(\vec{X}), a_{1}^{1}(\vec{X}),\left|d_{1}^{1}(\vec{X})\right|,\left|d_{1}^{1}(\vec{X})\right|\right\}$ and the features of series $\vec{Y}$ are $\overrightarrow{F_{2}(Y)}=\left\{a_{2}^{1}(\vec{X}),\left|d_{2}^{1}(\vec{X})\right|,\left|d_{2}^{2}(\vec{X})\right|,\left|d_{2}^{3}(\vec{X})\right|\right\}$. Comparing detail coefficients with approximation coefficients will induce errors and be meaningless.

To avoid this problem, we merge the distance of features within different appropriate scales. The distance of two features is replaced by the average of distance computed on two features with different appropriate scales. Suppose a time series $\vec{X}$ with appropriate scale $m$ and another time series $\vec{Y}$ with appropriate scale $n$, the distance between the appropriate features of $\vec{X}$ and $\vec{Y}$, $\operatorname{Disc}\left(\overrightarrow{F_{m}(X)}, \overrightarrow{F_{n}(Y)}\right)$, is defined as $\left(\operatorname{Disc}\left(\overrightarrow{F_{m}(X)}, \overrightarrow{F_{m}(Y)}\right)+\right.$ $\left.\operatorname{Disc}\left(\overrightarrow{F_{n}(X)}, \overrightarrow{F_{n}(Y)}\right)\right) / 2$. We can simply use distancebased classification and clustering algorithms for the proposed similarity strategy.

\subsubsection{1-NN Classification Algorithm Using the Proposed Similarity Strategy}

The classification algorithm is implemented by means of the 1-nearest neighbor algorithm (1-NN) [23], which we call WCANN (Wavelet Classification Algorithm based on 1-Nearest Neighbor). Table 1 shows an outline of the classification algorithm. The input is $S=$ $\left\{\overrightarrow{S_{1}}, \overrightarrow{S_{2}}, \ldots\right\}$ consists of a set of labeled time series data (the trained time series datasets) and $\vec{X}$ (a new emerged testing time series). The output is $x_{c}$, the label of $\vec{X} \cdot \vec{X}$ 
Table 1. The WCANN algorithm.

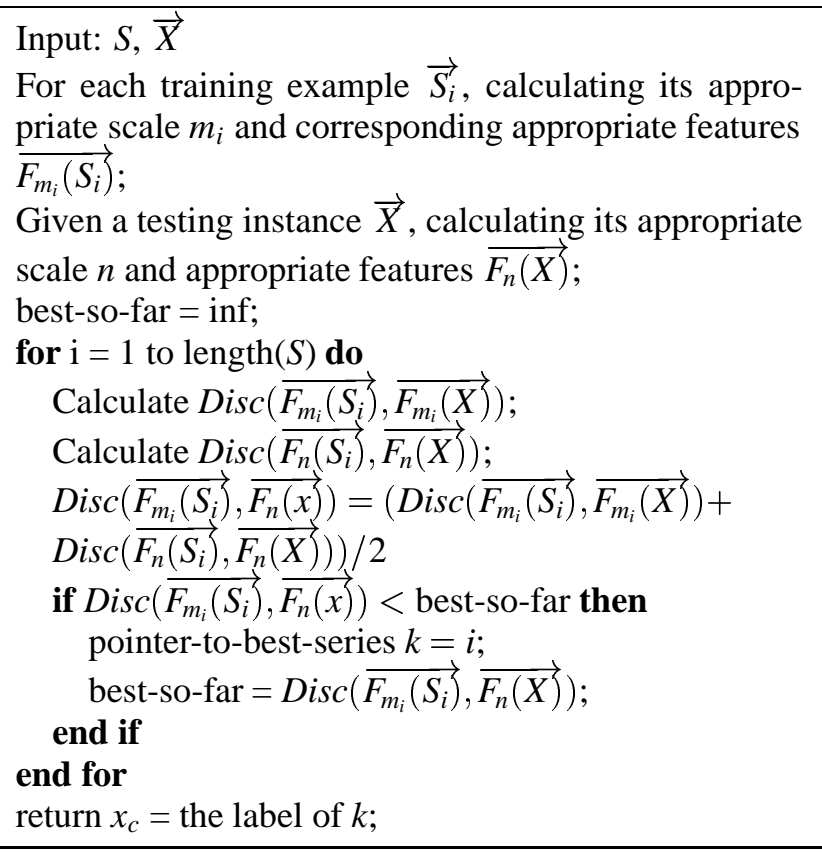

will be labeled as a member of a class if and only if, the distance between $\vec{X}$ and one instance of the class is smaller than between other instances.

The classification algorithm for noise reduced coefficients, WCANR (Wavelet Classification Algorithm with Noise Reduction), is similar to the WCANN algorithm. The only difference is that the noise within appropriate features is reduced before classification.

\subsubsection{Hierarchical Clustering Using the Proposed Simi- larity Strategy}

Clustering is one of the commonly used data mining tasks for discovering natural groups and identifying interesting patterns based on the similarity of data. Clustering is widely applied as a preprocessing procedure within a complex algorithm, it also can be used alone for describing the data.

Hierarchical clustering groups of data instances into a tree of clusters which is one of the best known clustering methods. The hierarchical clustering algorithms can be divided into agglomerative and divisive clustering, in terms of whether the hierarchical decomposition is carried in a bottom-up or top-down way [7]. As the computation of decomposition is normally simple for the agglomerative procedures, we only use agglomerative clustering algorithm with the proposed similarity strategy.

Given a set of $N$ time series to be clustered, we generate a $N \times N$ distance matrix consisted of the pair-distance between each of two time series using the defined similarity strategy. The basic process of agglomerative hierarchical clustering is described as below:

1. Assign each time series to its own cluster. For the given $N$ time series, we now have $N$ clusters, each containing just one time series. Let the distances between the clusters equal to the distances between the time series they contain.

2. Find the closest (most similar) pair of clusters and merge them into a single cluster, after that we have one less cluster.

3. Compute distances between the new cluster and each of the old clusters.

4. Repeat steps 2 and 3 until all items are clustered into a single cluster of size $N$.

\section{Experimental Evaluation}

To show the effectiveness of our approach, we performed experiments on five benchmark time series datasets. We compared the classification accuracy of our feature extraction algorithm with other feature extraction algorithms. All the feature extraction algorithms were compared with the one-nearest-neighbor algorithm $(1-N N)$, evaluated by leave-one-out cross validation. We also compared the clustering result of using features to that of using original time series. The single linkage is used for the agglomerative hierarchical clustering.

\subsection{Data Description}

We used five datasets (CBF, CC, Trance, Gun and Realitycheck) from the UCR Time Series Data Mining Archive [16]. As we need the label information, we only took the classified datasets for experiments. There are six classified datasets in the archive. The Auslan data is a multivariate dataset with which we can't apply our approach directly. All the other five datasets are used in our experiments. Realitycheck data only has one instance within each cluster that is too simple for classification, we only used it for clustering. The main features of the used data sets are described as below.

- Cylinder-Bell-Funnel (CBF): Contains three types of time series: cylinder (c), bell (b) and funnel (f). UCR Archive provides the source code for generating the samples. We generated 128 time series for each class with length 128 .

- Control Chart Time Series (CC): This data set has 100 instances for each of the six different classes of control charts.

- Trace dataset (Trace): The 4-class dataset contains 200 instances, 50 for each class. The dimensionality of the data is 275 .

- Gun Point dataset (Gun): The dataset has two classes, each containing 100 instances. The dimensionality of the data is 150 .

- Reality Check dataset (Realitycheck): This data set has 14 instances consists of data from different domains. The length of each time series is 1000 . 
Table 2. The error rates (\%) produced by various feature extraction methods with 1-NN classification algorithm for all four data sets.

\begin{tabular}{lcccc}
\hline Methods & CBF & CC & Gun & Trace \\
\hline WCANN & 0.26 & 0.67 & 4.5 & 7.5 \\
WCANR & 0.26 & 0.67 & 4.5 & 7.5 \\
WC-NN & 0.54 & 1.73 & 7.25 & 12.39 \\
DFT-NN & 0.41 & 2.52 & 6.48 & 10.84 \\
SVD-NN & 0.61 & 2.29 & 5.99 & 12.37 \\
NN & 0.26 & 1.33 & 5.5 & 11 \\
\hline
\end{tabular}

\subsection{Classification Results}

We only used Euclidean distance for all the 1- $N N$ algorithms in our experiments because it is the most commonly used distance measures. Note that Dynamic Time Warping (DTW) as a similarity measure can achieve very high accuracy (even no error) for some time series datasets [27]. However, one of the well-known drawbacks of DTW is that it needs quadratic time and space complexity that is only useful for short time series. The accuracy of the classified results is measured by error rates. We compared six different feature extraction algorithms with 1-NN for the CBF, CC, Trace and Gun data sets described above. WCANN and WCANR are our proposed algorithms described in section 3.5. NN is the 1-nearest neighbor algorithm that uses the raw data [17]. WC-NN is the algorithm that extracts first few Haar wavelet coefficients as features [3]. DFT-NN is the algorithm using first few Fourier coefficients as features [26]. SVD-NN uses Singular Value Decomposition (SVD) to generate a low-rank matrix for approximating the whole set of time series [20]. As WC-NN, DFT-NN and SVD-NN don't give the solution for choosing feature dimensionality, we take the average error rates with all possible feature dimensions produced by these algorithms. Table 2 gives the error rates produced by various feature extraction algorithms with 1-NN classification algorithm.

The WCANN algorithm achieves the same classification accuracy as the WCANR algorithm on all the four data sets. Noise reduction upon the extracted appropriate features doesn't affect the classification result, i.e., the appropriate features are robust against noise in terms of classification. WCANN and WCANR take the same classification accuracy with NN algorithm on CBF data and higher accuracy on other three datasets. WCANN and WCANR algorithm outperform WC-NN, DFT-NN and SVD-NN on classification accuracy for all the datasets used.

\subsection{Clustering}

Hierarchical clustering is good for comparing different data representation by visually observing the dendrogram of the clustering tree [17]. For time series data, the intuitive feeling of the natural time series groups can be used as the clustering quality measure. Fig. 2 shows the dendrogram produced by agglomerative hierarchical clustering

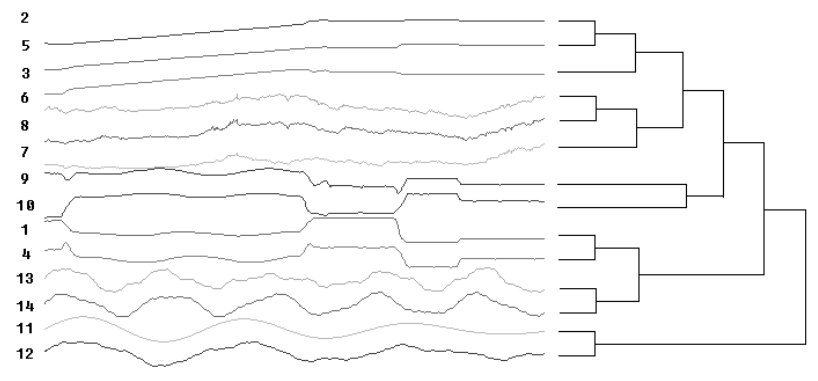

Fig. 2. The dendrogram generated by agglomerative clustering algorithm for original Realitycheck data set, single linkage is used.

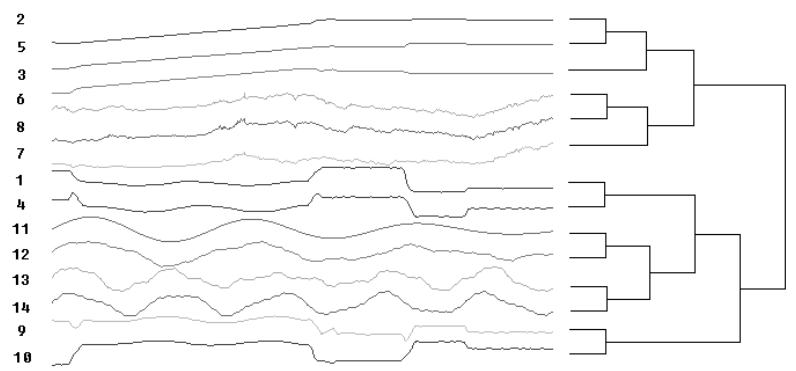

Fig. 3. The dendrogram generated by agglomerative clustering algorithm with the features of Realitycheck data set, single linkage is used.

algorithm with the original time series. The extracted features and noise-reduced features produce the same clustering results and the results are shown in Fig.3. The extracted features are not affected by the noise during the clustering process for the used datasets. The extracted features are robust against noise in terms of clustering. The subgroup contains instances $\{11,12\}$ and the subgroup contains instances $\{13,14\}$ are similar. They are successfully clustered into one subgroup with our defined features shown in Fig.3 and are not in the same subgroup with the original time series shown in Fig.2.

\section{Conclusions}

We exploited the multi-scale property of Haar wavelet transform to extract features combining the global information and partial information together. We proposed a method for automatically choosing the appropriate features by the concentration of the feature coefficients. We proposed a distance strategy for the appropriate features with which distance-based classification and clustering algorithms can be easily applied. We conducted experiments on several widely used time series datasets and compared the classification results of our algorithms and those of other four algorithms. Our algorithms outper- 
formed other four algorithms on classification accuracy. We also compared the clustering results produced by the appropriate features and the original time series. The appropriate features could create more natural groupings of the data. The experimental results showed that the appropriate features always produce the same classification and clustering results with noise-reduced appropriate features. It indicates that several important coefficients within the appropriate features dominate the classification and clustering processes. The extracted appropriate features are robust against noise in terms of classification and clustering.

\section{Acknowledgements}

The authors thank Prof. Keogh for providing the experimental data. The authors thank two anonymous reviewers for suggestions that markedly clarified and improved the paper.

\section{References:}

[1] R. Agrawal, C. Faloutsos, and A. Swami, "Efficient Similarity Search in Sequence Databases," In Proceedings of the 4th Conference on Foundations of Data Organization and Algorithms, pp. 6984, October, 1993.

[2] C. S. Burrus, R. A. Gopinath, and H. Guo, "Introduction to Wavelets and Wavelet Transforms, A Primer," Prentice Hall, Englewood Cliffs, NJ, 1997.

[3] K. Chan, A. W. Fu, and T. Y. Clement, "Harr Wavelets for Efficient Similarity Search of Time-Series: with and without Time Warping," IEEE Trans. on Knowledge and Data Engineering, 15(3): pp. 686$705,2003$.

[4] R. R. Coifman, and M. V. Wickerhauser, "Entropy-Based Algorithms for Best Basis Selection," IEEE Trans. on Information Theory, 38(2): pp. 713-718, 1992.

[5] D. L. Donoho, "De-noising by soft-thresholding," IEEE Trans. on Information Theory, 41(3): pp. 613-627, 1995

[6] D. L. Donoho, and I. M. Johnson, "Ideal spatial adaptation via wavelet shrinkage," Biometrika, 81: pp. 425-455, 1994.

[7] R. Duda, P. Hart, and D. Stork, "Pattern Classification," John Wiley \& Sons, New York, second edition, 2001.

[8] M. Gavrilov, D. Anguelov, and P. Indyk, "Mining the Stock Market: Which Measure is Best?," In Proceedings of The 6th ACM SIGKDD International Conference on Knowledge Discovery and Data Mining, pp. 487-496, August, 2000.

[9] P. Geurts, "Pattern Extraction for Time Series Classification," In Proceedings of the Principles of Data Mining and Knowledge Discovery, 5th European Conference, pp. 115-127, September, 2001.

[10] T. B. Ho, T. D. Nguyen, S. Kawasaki, S. Q. Le, D. D. Nguyen, H. Yokoi, and K. Takabayashi, "Mining Hepatitis Data with Temporal Abstraction," In Proceedings of the 9th ACM International Conference on Knowledge Discovery and Data Mining, pp. 369-377, August, 2003.

[11] D. Jiang, C. Tang, and A. Zhang, "Cluster Analysis for Gene Expression Data: A Survey," IEEE Trans. on Knowledge and Data Engineering, 16(11): pp. 1370-1386, 2004.

[12] X. Jin, Y. Lu, and C. Shi, "Similarity Measure Based on Partial Information of Time Series," In Proceedings of The 8th ACM SIGKDD International Conference on Knowledge Discovery and Data Mining, pp. 544-549, August, 2002.

[13] M. W. Kadous, "Learning Comprehensible Descriptions of Multivariate Time Series," In Proceedings of the 6th International Conference on Machine Learning, pp. 454-463, September, 1999.

[14] T. Kalayci, and O. Ozdamar, "Wavelet Preprocessing for Automated Neural Network Detection of EEG Spikes," IEEE Eng. in Medicine and Biology, 14: pp. 160-166, 1995.

[15] E. Keogh, K. Chakrabarti, M. Pazzani, and S. Mehrotra, "Dimensionality Reduction of Fast Similarity Search in Large Time Series Databases," Journal of Knowledge and Information System, 3: pp. 263-286, 2000

[16] E. Keogh, and T. Folias. "The UCR Time Series Data Mining Archive," http://www.cs.ucr.edu/ eamonn/TSDMA/index.html, 2002.

[17] E. Keogh, and S. Kasetty, "On the Need for Time Series Data Mining Benchmarks: A Survey and Empirical Demonstration," Data Mining and Knowledge Discovery, 7(4): pp. 349-371, 2003.
[18] E. Keogh, S. Lonardi, and C. A. Ratanamahatana, "Towards Parameter-Free Data Mining," In Proceedings of the 10th ACM SIGKDD International Conference on Knowledge Discovery and Data Mining, pp. 206-215, August, 2004.

[19] E. Keogh, and M. Pazzani, "An Enhanced Representation of Time Series which Allows Fast and Accurate Classification, Clustering and Relevance Feedback," In Proceedings of the 4th International Conference of Knowledge Discovery and Data Mining, pp. 239241, August, 1998.

[20] F. Korn, H. Jagadish, and C. Faloutsos, "Efficiently Supporting ad hoc Queries in Large Datasets of Time Sequences," In Proceedings of The ACM SIGMOD International Conference on Management of Data, pp. 289-300, May, 1997.

[21] S. Lawrence, A. Back, A. Tsoi, and C. L. Giles, "The Gamma MLP - Using Multiple Temporal Resolutions for Improved Classification," In Proceedings of the 1997 IEEE Workshop on Neural Networks for Signal Processing VII, pp. 256-265, Piscataway, NJ, 1997, IEEE Press.

[22] J. Lin, E. Keogh, S. Lonardi, and B. Chiu, "A Symbolic Representation of Time Series, with Implications for Streaming Algorithms," In Proceedings of the 8th ACM SIGMOD Workshop on Research Issues in Data Mining and Knowledge Discovery, pp. 2-11, June, 2003.

[23] T. Mitchell, "Machine Learning," McGraw-Hill, New York, 1997.

[24] S. Pitter, and S. V. Kamarthi, "Feature Extraction From Wavelet Coefficients for Pattern Recognition Tasks," IEEE Trans. on Pattern Recognition and Machine Intelligence, 21(1): pp. 83-85, January, 1999.

[25] I. Popivanov, and R. J. Miller, "Similarity Search over Time-Series Data Using Wavelets," In Proceedings of The 18th International Conference on Data Engineering, pp. 212-221, Feburary, 2002.

[26] D. Rafiei, and A. Mendelzon, "Efficient Retrieval of Similar Time Sequences Using DFT," In Proceedings of the 5th International Conference on Foundations of Data Organizations, pp. 249-257, 1998.

[27] C. A. Ratanamahatana, and E. Keogh, "Three Myths about Dynamic TimeWarping," In SIAM International Conference on Data Mining, Newport Beach, CA, April, 2005.

[28] J. F. Roddich, and M. Spiliopoulou, "A Survey of Temporal Knowledge Discovery Paradigms and Methods," IEEE Trans. on Knowledge and Data Engineering, 14(4): pp. 750-767, 2002.

[29] C. Shahabi, X. Tian, and W. Zhao, "TSA-Tree: A Wavelet-Based Approach to Improve the Efficiency of Multi-Level Surprise and Trend Queries on Time-Series Data," In Proceedings of the 12th International Conference on Scientific and Statistical Database Management, pp. 55-68, July, 2000.

[30] C. E. Shannon, "A Mathematical Theory of Communication," Bell System Technical Journal, 27: pp. 379-423, 1948.

[31] Z. R. Struzik, and A. Siebes, "The Harr Wavelet Transform in the Time Series Similarity Paradigm," In Proceedings of the 3rd European Conference on Principles of Data Mining and Knowledge Discovery, pp. 12-22, September, 1999.

[32] I. N. Tansel, C. Mekdeci, and C. McLaughlin, "Detection of Tool Failure in End Milling with Wavelet Transformations and Neural Networks (WT-NN)," International Journal of Machine Tolls and Manufacture, 35(8): pp. 1137-1147, 1995.

[33] Y. Yamada, E. Suzuki, H. Yokoi, and K. Takabayashi, "DecisionTree Induction from Time-Series Data Based on Standard-Example Split Test," In Proceedings of the 20th International Conference on Machine Learning (ICML03), pp. 840-847, August, 2003.

[34] B. K. Yi, and C. Faloustos, "Fast Time Sequence Indexing for arbitrary $L_{p}$ norms," In Proceedings of the 26th International Conference on Very Large Databases, pp. 385-394, September, 2000. 


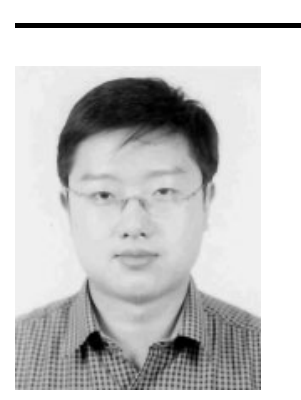

Name:

Hui Zhang

\section{Affiliation:}

School of Knowledge Science, Japan Advanced Institute of Science and Technology (JAIST)

\section{Address:}

1-1 Asahidai, Nomi, Ishikawa 923-1292, Japan

\section{Brief Biographical History:}

1993- B.S., Southwest University of Science and Technology, China 2000- Master, Wuhan University of Science and Technology, China Main Works:

- "Finding the Clustering Consensus of Time series with Multi-Scale Transform," In Proceedings of the Fourth IEEE International Workshop on Soft Computing as Transdisciplinary Science and Technology, pp. 1081-1090, Springer-Verlag, 2005.

- "Blind feature extraction for time-series classification using Haar wavelet transform," In Proceedings of the Second International Symposium on Neural Networks, pp. 605-610, Springer-Verlag, 2005.

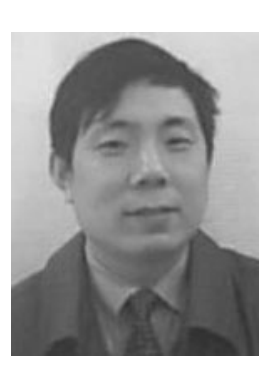

Name:

Mao-Song Lin

\section{Affiliation:}

Associate Professor, School of Computer Science, Southwest University of Science and Technology, China

\section{Address:}

Mianyang, Sichuan, 621002, China

\section{Brief Biographical History:}

2000- Associate Professor, Southwest University of Science and Technology, China

\section{Main Works:}

- "A Non-parametric Wavelet Feature Extractor for Time series Classification," In Proceedings of the 8th Pacific-Asia Conference on Knowledge Discovery and Data Mining, pp. 595-603, Springer-Verlag, 2004.

- "An Evolutionary K-Means Algorithm for Clustering Time Series Data," In Proceedings of the Third International Conference on Machine Learning and Cybernetics, pp. 1282-1287, IEEE Press, 2004.

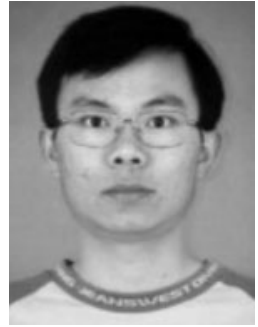

Name:

Wei Huang

\section{Affiliation:}

School of Knowledge Science, Japan Advanced Institute of Science and Technology (JAIST)

\section{Address:}

1-1 Asahidai, Nomi, Ishikawa 923-1292, Japan

\section{Brief Biographical History:}

2000- B.S., University of Science and Technology of China

2005- Ph.D., Japan Advanced Institute of Science and Technology

Main Works:

- "Mining Scientific Literature to Predict New Relationships," Intelligent Data Analysis, Vol.9, No.2, pp. 219-234, 2005.

- "Forecasting Foreign Exchange Rates with Artificial Neural Networks: A Review," International Journal of Information Technology and Decision Making, Vol.3, No.1, pp. 145-165, 2004. 